CMEARTICLE

\title{
Clinics in diagnostic imaging (162)
}

Dinesh $R$ Singh $^{1}$, MMed, FRCR, Geoiphy G Pulickal ${ }^{1}$, MMed, FRCR, Zhiwen J LO ${ }^{2}$, MBBS, BMed, Wilfred CG Peh${ }^{1}$, FRCP, FRCR

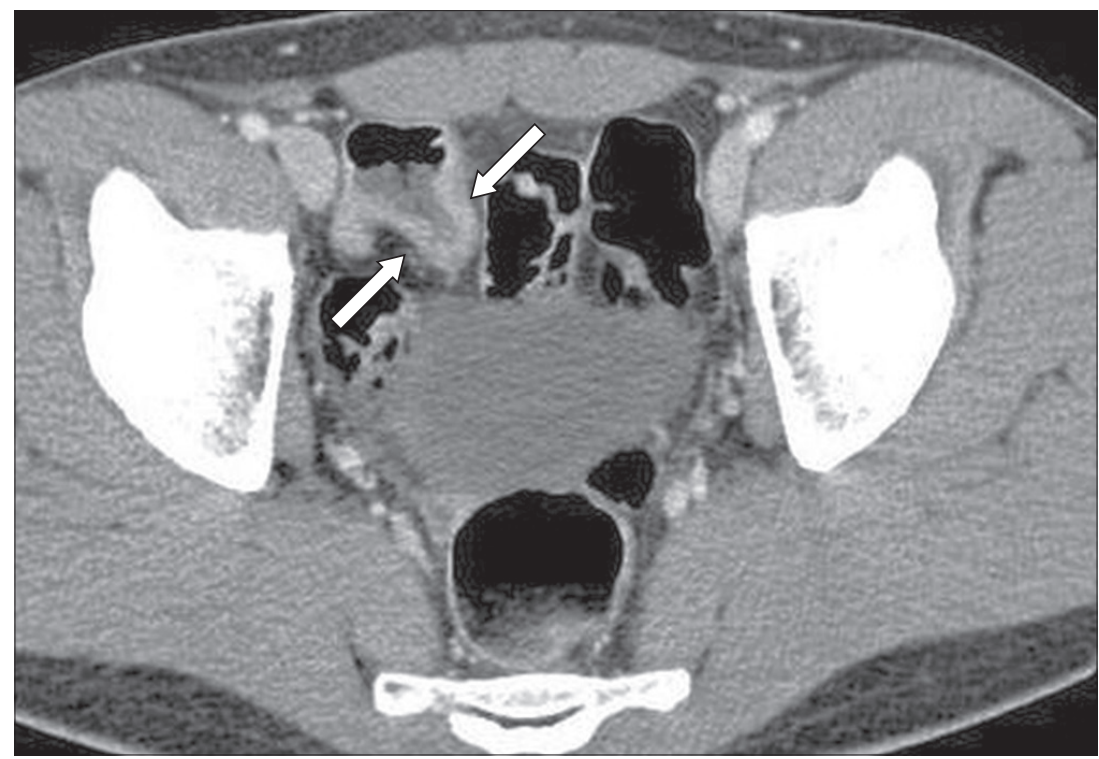

Fig. 1 Contrast-enhanced axial CT image.
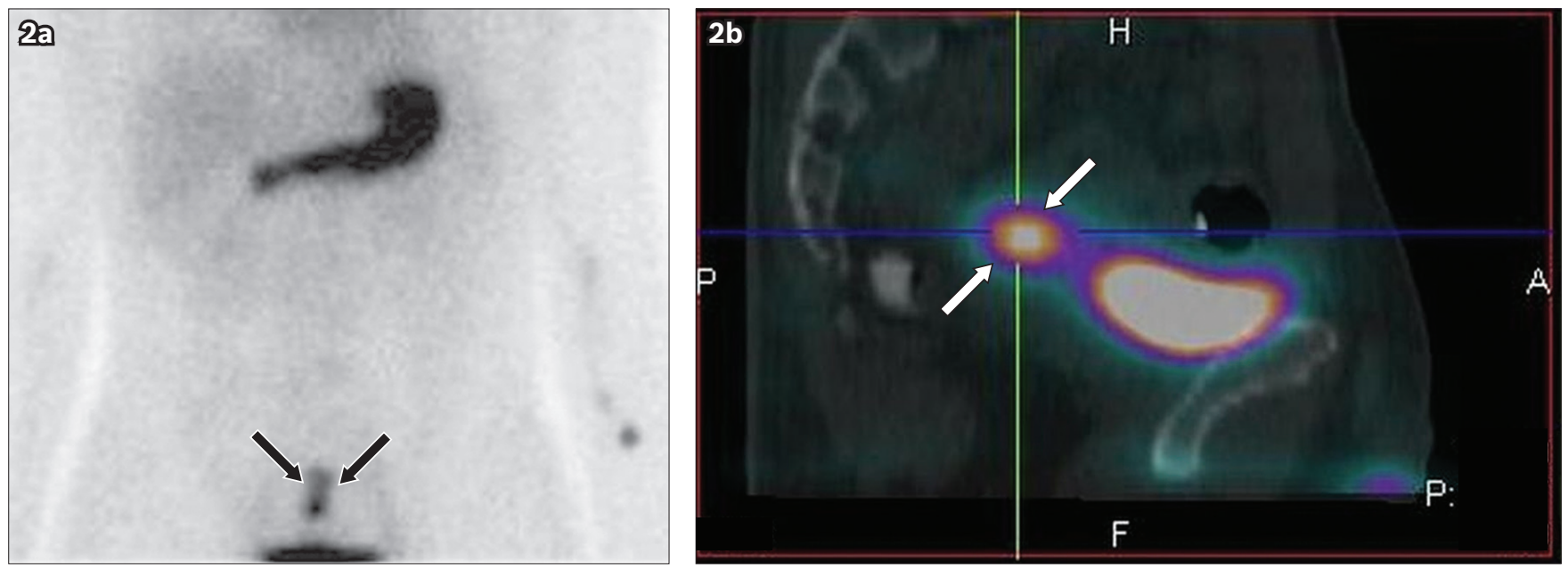

Fig. 2 Tc-99m pertechnetate (a) anterior planar scintigraphy and (b) sagittal single-photon emission computed tomography (SPECT)/CT fusion images.

\section{CASE PRESENTATION}

A 28-year-old Chinese man presented to the accident and emergency department with active bleeding per rectum. The patient had experienced similar bouts of bleeding in the preceding two years and was treated symptomatically. He was stable on clinical examination and had normal blood pressure, pulse rate and respiratory rate. Although there was altered blood on digital rectal examination, no masses were felt. Computed tomography (CT) was performed to identify the possible cause of bleeding (Fig. 1). Nuclear medicine scintigraphy using technetium (Tc)$99 \mathrm{~m}$ pertechnetate was performed the following day to obtain anterior planar (Fig. 2a) and single-photon emission computed tomography (SPECT)/CT fusion (Fig. 2b) images. What do the CT and scintigraphy images show? What is the diagnosis? 


\section{IMAGE INTERPRETATION}

Contrast-enhanced axial CT image (Fig. 1) shows posterior outpouching from a segment of the distal ileum (arrows) associated with mild wall thickening and increased mucosal enhancement. There is congestion of the surrounding mesentery with soft tissue stranding. No extraluminal air to suggest perforation is observed. Tc-99m pertechnetate anterior planar scintigraphy (Fig. 2a) and SPECT/CT fusion (Fig. 2b) images show focal uptake in the right hemipelvis (arrows) superior and posterior to the urinary bladder. This uptake gradually increased over time (not shown). The location of the focal uptake corresponded with the distal ileum outpouching.

\section{DIAGNOSIS}

Meckel's diverticulum.

\section{CLINICAL COURSE}

The imaging diagnosis was confirmed on retrograde singleballoon endoscopy, during which features of melanosis coli were seen. The distal ileum, from which biopsy samples were obtained, showed areas of scarring and multiple ulcerations. Subsequent laparoscopic evaluation confirmed the presence of the Meckel's diverticulum, which arose about $62 \mathrm{~cm}$ from the ileocaecal junction. Small bowel resection was performed to incorporate the diverticulum and the areas of ulceration. The resected bowel, which measured $28 \mathrm{~cm}$ in length and included a $4.5-\mathrm{cm}$ long outpouching from the antimesenteric surface (Fig. 3), was sent for histopathological evaluation. The diverticulum contained ectopic gastric and pancreatic tissue; however, there were no signs of inflammation, dysplasia or malignancy.

\section{DISCUSSION}

Meckel's diverticulum is the most common congenital anomaly of the gastrointestinal tract and is found in about $2 \%$ of the general population. ${ }^{(1,2)}$ It was first described in 1809 by Johann Friedrich Meckel and is slightly more common in men. ${ }^{(2)}$ It contains all three layers of the intestinal wall and hence is a true diverticulum. Originating from the antimesenteric border of the distal ileum, it arises due to the incomplete closure of the omphalomesenteric duct. In the majority of cases, this diverticulum is located about $100 \mathrm{~cm}$ from the ileocaecal valve. This diverticulum can vary in length and width, to as long as $10 \mathrm{~cm}$ in some instances. Meckel's diverticulum can contain heterotopic mucosa, commonly the gastric or pancreatic mucosa. ${ }^{(1,2)}$ Heterotopic gastric mucosa is more common than duodenal or jejunal mucosa and can result in ulcerations, which can subsequently cause gastrointestinal bleeding. Vascular supply to the diverticulum is mostly from the omphalomesenteric artery, which arises from one of the ileal branches of the superior mesenteric artery.

Meckel's diverticulum usually remains asymptomatic throughout life; however, it can also present with a number of well-known complications. These complications may range from varying degrees of inflammation, gastrointestinal tract bleeding ${ }^{(3-5)}$ and small bowel obstruction ${ }^{(6)}$ to perforation. Patients often present with acute abdominal pain localised to the right lower quadrant.

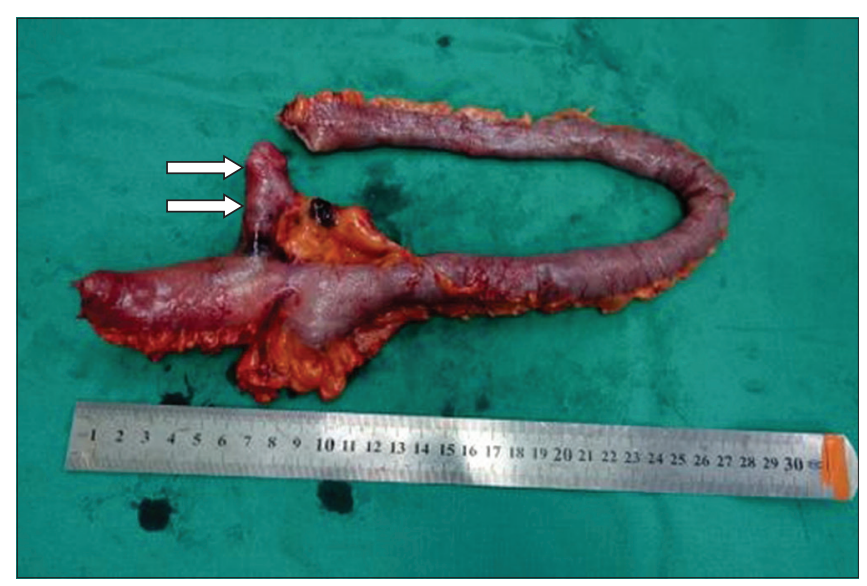

Fig. 3 Photograph of the resected specimen shows a short length of ileum with posterior outpouching (arrows), consistent with a Meckel's diverticulum.

The estimated lifetime risk of complications in patients with this diverticulum is $4 \% .{ }^{(1)}$ Gastrointestinal haemorrhage is a serious presentation that is more common and severe in children. ${ }^{(7)}$ The bleeding is due to heterotopic gastric mucosa, which results in ulcerations also seen in the adjacent loops of ileum. Another common complication is acute diverticulitis, which may be of varying degrees of severity. The orifice of the diverticulum may be blocked by an enterolith or retained foreign bodies, ${ }^{(8)}$ resulting in stasis and infection. The diverticulum may also undergo torsion, resulting in inflammation or ischaemia. Acute inflammation of a Meckel's diverticulum is a well-known mimic of acute appendicitis. Severe cases of inflammation may result in perforation, which is a life-threatening complication. Many other conditions involving the lower abdomen and pelvis can also present with complaints similar to those of Meckel's diverticulum, thus making Meckel's diverticulum extremely difficult to diagnose based on clinical features alone. Small bowel obstruction is also a well-known complication and can occur due to the twisting of the bowel at the site of the diverticulum. Larger diverticula have greater chances of causing obstruction and may also be associated with intussusceptions. ${ }^{(9)}$ Although rarely seen, a large Meckel's diverticulum may invert within the distal ileum and cause obstruction. Carcinoid tumours arising within Meckel's diverticula are rare, ${ }^{(10)}$ although they are the most commonly associated neoplasm. Malignant neoplasms arising from the diverticulum are extremely rare.

Imaging plays an extremely important role in evaluating patients with suspected Meckel's diverticulum. Radiographs of the abdomen are usually unremarkable unless there are features of intestinal obstruction or perforation. Ultrasonography is often performed in children as the first imaging modality. However, the diagnosis is extremely difficult; Meckel's diverticulum is similar in appearance to the appendix, although it is in a different location. The diverticulum is visualised as a blind-ending, tubular echogenic structure that has clear communication with a small bowel loop. Contrast-enhanced CT of the abdomen plays a very important role in diagnosis and can also identify associated complications in the majority of cases. The diverticulum is visualised as a blind-ending tubular structure arising from the 


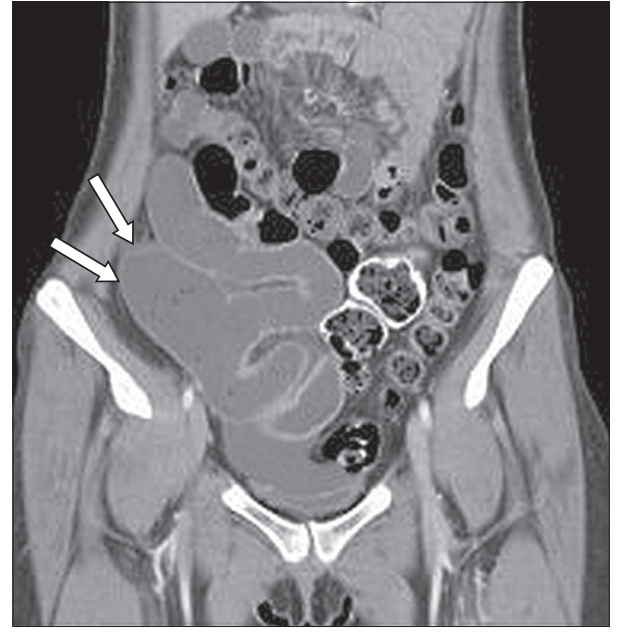

Fig. 4 A 35-year-old woman was evaluated for left iliac fossa pain. Contrast enhanced coronal CT image shows a large blind-ending tubular structure arising from the small bowel (arrows). This incidental finding is a Meckel's diverticulum.

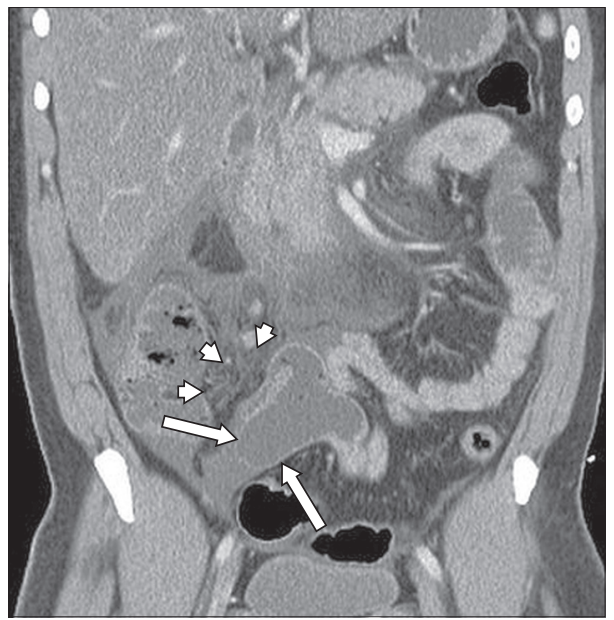

Fig. 6 A 40-year-old Chinese man presented with acute onset severe lowe abdominal pain. Contrast-enhanced coronal CT image shows an inflamed Meckel's diverticulum (arrows) with surrounding free fluid and fat stranding (arrowheads), typical of diverticulitis.

antimesenteric border of the ileum, a short distance proximal to the ileocaecal junction (Fig. 4). The diverticulum may show intraluminal fluid, air, faeces or even enteroliths. There may be resultant obstruction with proximal bowel dilatation (Fig. 5). Increased mucosal enhancement and wall thickening are signs of inflammation, while poor mucosal enhancement suggests ischaemia. Acute diverticulitis is identified by increased mucosal enhancement, wall thickening, surrounding soft-tissue stranding and fluid (Fig. 6). Severe inflammation or ischaemia can result in perforation with evidence of localised or generalised free air in the peritoneal cavity (Fig. 7). Associated small bowel obstruction necessitates careful evaluation of the transition zone to identify the presence of the diverticulum. CT mesenteric angiography may be performed to identify any active contrast leak in patients presenting with gastrointestinal haemorrhage. Tumours in Meckel's diverticulum have nonspecific imaging features and are visualised as intraluminal or intramural enhancing soft tissue. It is important to identify any involvement of the adjacent small bowel loops, as they are markers for malignant change.
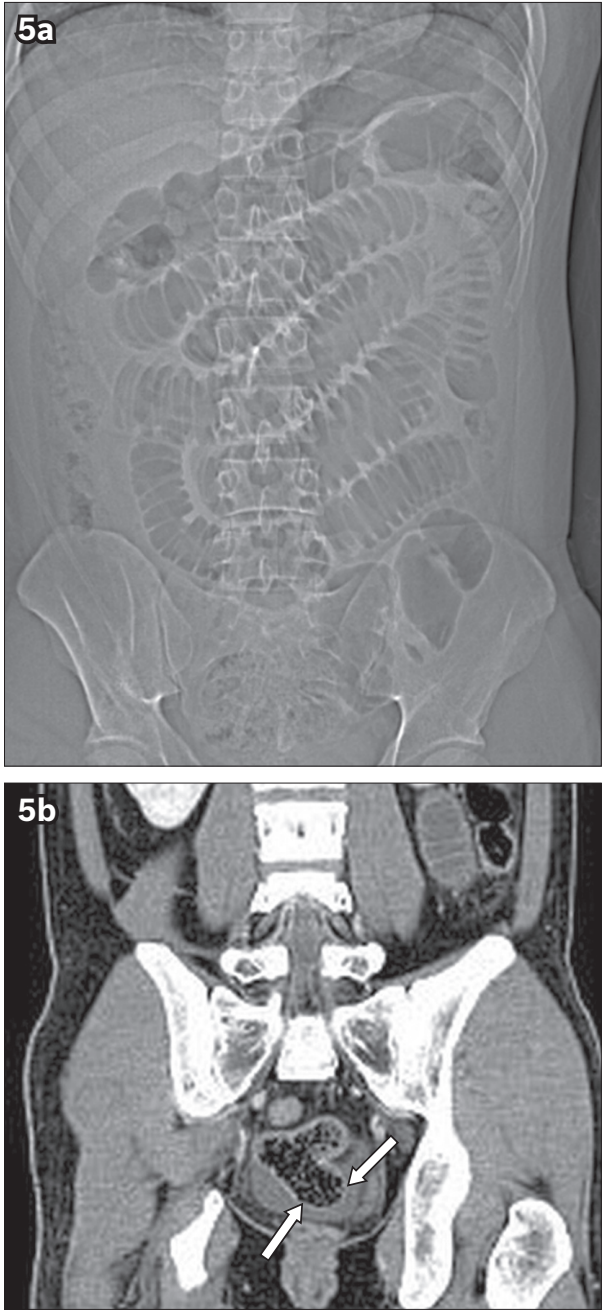

Fig. 5 A 25-year-old man presented with colicky right iliac fossa pain of one-day duration. (a) Coronal CT scout image shows multiple grossly dilated small bowel loops. (b) Contrast-enhanced coronal CT image shows faecal-laden distal ileum and a Meckel's diverticulum (arrows).

Angiographic studies may also be performed in cases of severe gastrointestinal bleeding and may help in identifying the persistent omphalomesenteric artery. However, this is difficult and may require selective catheterisation of the distal ileal arteries, as the omphalomesenteric artery most commonly originates from the mid-distal branches of the superior mesenteric artery.

Nuclear medicine scintigraphy also plays an important role in identifying Meckel's diverticulum; however, its role is limited to cases with heterotopic gastric mucosa. ${ }^{(11)}$ Overall diagnostic accuracy in these cases may be up to $90 \%$. $^{(1)}$ The overall sensitivity of Tc-99m pertechnetate scintigraphy is about $60 \%$ in cases of Meckel's diverticulum. The uptake of pertechnetate by the ectopic gastric mucosa in the diverticulum is focal and simultaneous with the uptake of pertechnetate by the stomach, and gradually increases on subsequent phases of imaging. It is important to correlate the scintigraphic findings with CT imaging, as many of these cases are a diagnostic challenge.

Timely diagnosis of a complicated Meckel's diverticulum is very important, as the management varies depending on the severity of the complications. Surgery is usually indicated in cases of symptomatic diverticula, especially those presenting with 

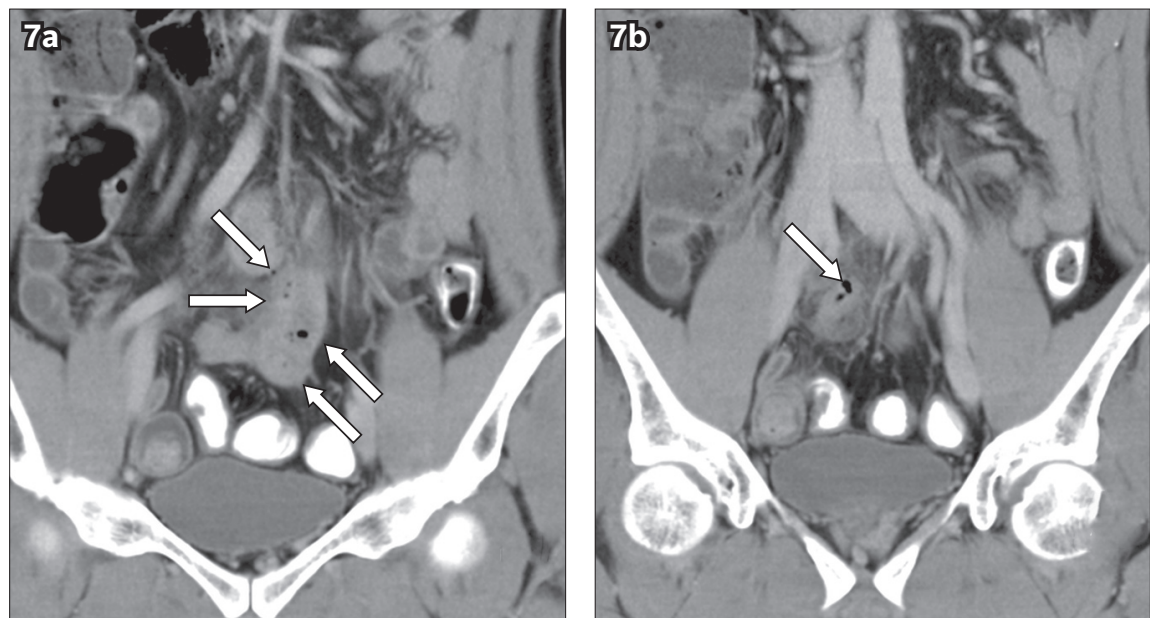

Fig. 7 A 49-year-old man complained of acute onset epigastric discomfort. (a) Contrast-enhanced coronal CT image shows an outpouching from the distal ileum (arrows) with pockets of free air in the small bowel mesentery (arrowheads), consistent with perforation. (b) Contrast-enhanced coronal CT image shows the inflamed Meckel's diverticulum (arrow).

bleeding. The decision for surgery also depends on the overall condition of the patient. During surgery, it is important to resect all foci of the ectopic mucosa and look for associated ulcerations, which are typically seen at the junction of the normal and ectopic mucosa. Asymptomatic patients are conservatively managed and surgery is usually not performed. Meckel's diverticula can also be incidentally detected during laparotomy and their treatment is controversial. The choice of resection in such cases depends on the surgeon, overall condition of the patient and extent of the surgery being performed.

In summary, Meckel's diverticulum is a well-known cause of right lower quadrant pain, especially in young patients. The

ABSTRACT A 28-year-old Chinese man presented with acute bleeding per rectum. Computed tomography showed a posterior outpouching arising from the distal ileum. The outpouching had hyperaemic walls, but no active contrast extravasation was detected. Technetium-99m pertechnetate scintigraphy showed focal areas of abnormal uptake in the right side of the pelvis, superior and posterior to the urinary bladder. These areas of uptake appeared simultaneously with the gastric uptake and demonstrated gradual increase in intensity on subsequent images. The diagnosis of Meckel's diverticulum was confirmed on surgery and the lesion was resected. The clinical and imaging features of Meckel's diverticulum are discussed.

Keywords: acute abdomen, diverticulitis, gastrointestinal bleeding, Meckel's diverticulum clinical presentation can often overlap with a number of other pathologies, making the diagnosis difficult. Contrast-enhanced $\mathrm{CT}$, in conjunction with Tc-99m pertechnetate scintigraphy, can aid radiologists in making a confident diagnosis. Symptomatic patients usually require early surgery to resect the diverticulum.

\section{REFERENCES}

1. Elsayes KM, Menias CO, Harvin HJ, Francis IR. Imaging manifestations of Meckel's diverticulum. AJR Am J Roentgenol 2007; 189:81-8.

2. Bennett GL, Birnbaum BA, Balthazar EJ. CT of Meckel's diverticulitis in 11 patients. AJR Am J Roentgenol 2004; 182:625-9.

3. Rashid OM, Ku JK, Nagahashi M, Yamada A, Takabe K. Inverted Meckel's diverticulum as a cause of occult lower gastrointestinal hemorrhage. World J Gastroenterol 2012; 18:6155-9.

4. Kalla R, McGrath S, Campbell S. An unusual cause of gastrointestinal bleeding: there is more than meets the eye. Gastroenterology 2013; 145:729, 915

5. Vaughan-Shaw PG, Dwerryhouse S. Life-threatening gastrointestinal haemorrhage from a large Meckel's diverticulum. BMJ Case Rep 2013; 2013.

6. Jo JH, Seo KW, Yoon KY. [Loop formation of Meckel's diverticulum causing intestinal obstruction]. Korean J Gastroenterol 2014; 63:56-8. Korean.

7. Borowski DW, Hobday C, Agarwal AK. Haemoperitoneum caused by perforation of Meckel's diverticulum in a 5-year-old child. BMJ Case Rep 2010; 2010 .

8. Bingham JR, Causey MW, Haque MI. Phytobezoar within Meckel's diverticulum: an unusual cause of intestinal obstruction. Am Surg 2014; 80:E94-6.

9. Lee TH, Kim JO, Kim JJ, et al. A case of intussuscepted Meckel's diverticulum. World J Gastroenterol 2009; 15:5109-11.

10. Lorenzen AW, O'Dorisio TM, Howe JR. Neuroendocrine tumors arising in Meckel's diverticula: frequency of advanced disease warrants aggressive management. J Gastrointest Surg 2013; 17:1084-91.

11. Shammas A, Vali R, Charron M. Pediatric nuclear medicine in acute care. Semin Nucl Med 2013; 43:139-56. 


\section{SINGAPORE MEDICAL COUNCIL CATEGORY 3B CME PROGRAMME} (Code SMJ 201509B)

Question 1. Regarding Meckel's diverticulum:
(a) It is a true diverticulum.
(b) It contains only two layers of the intestinal wall.
(c) It originates from the distal jejunum.
(d) It arises from the antimesenteric border of the distal ileum.

Question 2. Regarding Meckel's diverticulum:
(a) It arises due to incomplete closure of the omphalomesenteric duct.
(b) It is always $10 \mathrm{~cm}$ in length.
(c) It may contain heterotopic gastric mucosa.
(d) It never contains pancreatic mucosa.

Question 3. Regarding complications associated with Meckel's diverticulum:
(a) It is usually asymptomatic.
(b) Gastrointestinal haemorrhage is more common in adults.
(c) The estimated lifetime risk of complications in patients with the diverticulum is $20 \%$.
(d) The diverticulum may undergo torsion.

Question 4. Regarding imaging of Meckel's diverticulum:
(a) Ultrasonography evaluation has no role.
(b) Radiographs are usually unremarkable.
(c) Computed tomography (CT) evaluation has limited role.
(d) Technetium-99m pertechnetate scintigraphy is especially useful in cases with heterotopic gastric mucosa.

Question 5. Regarding CT imaging of Meckel's diverticulum:
(a) It is visualised as a blind-ending tubular structure.
(b) Increased mucosal enhancement is a sign of inflammation.
(c) Wall thickening is a sign of inflammation.
(d) Presence of intraluminal soft tissue is suspicious for underlying neoplasm.

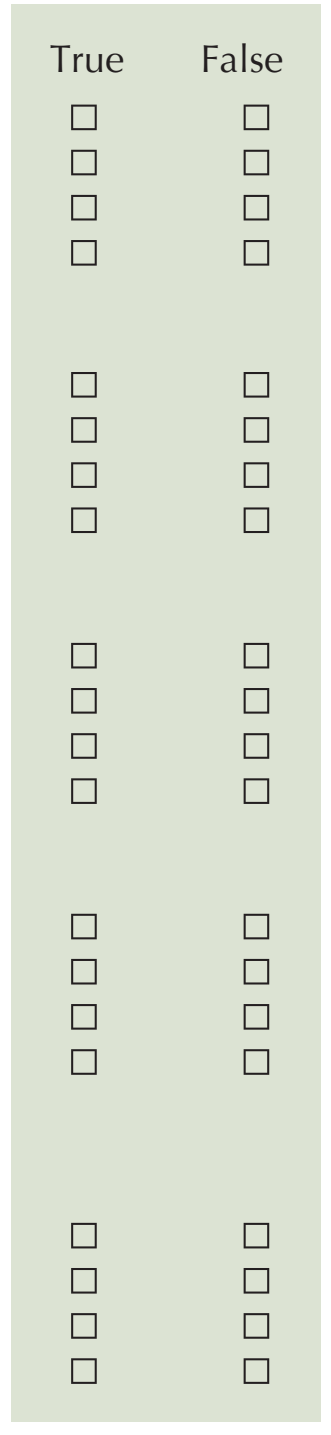

\footnotetext{
Doctor's particulars:

Name in full

MCR number

Email address

Specialty:

\section{SUBMISSION INSTRUCTIONS:}

(1) Log on at the SMJ website: http://www.sma.org.sg/publications/smjcurrentissue.aspx and select the appropriate set of questions. (2) Provide your name, email address and MCR number. (3) Select your answers and click "Submit".

RESULTS:

(1) Answers will be published in the SMJ November 2015 issue. (2) The MCR numbers of successful candidates will be posted online at the SMJ website by 2 November 2015. (3) Passing mark is $60 \%$. No mark will be deducted for incorrect answers. (4) The SMJ editorial office will submit the list of successful candidates to the Singapore Medical Council. (5) One CME point is awarded for successful candidates.

Deadline for submission: (September 2015 SMJ 3B CME programme): 12 noon, 26 October 2015.
} 